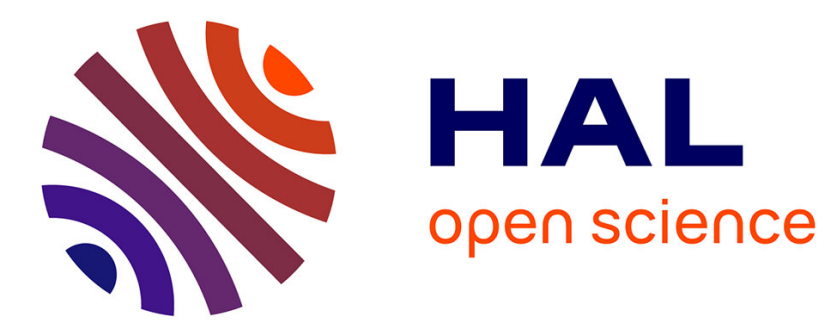

\title{
Réflexions sur le concept de détachement adolescentaire
}

\author{
M. Delhaye, C. Kempenaers, J. Burton, L. Goossens, P. Linkowski
}

\section{To cite this version:}

M. Delhaye, C. Kempenaers, J. Burton, L. Goossens, P. Linkowski. Réflexions sur le concept de détachement adolescentaire. Annales Médico-Psychologiques, Revue Psychiatrique, 2011, 169 (4), pp.215. 10.1016/j.amp.2010.07.004 . hal-00748150

\section{HAL Id: hal-00748150 \\ https://hal.science/hal-00748150}

Submitted on 5 Nov 2012

HAL is a multi-disciplinary open access archive for the deposit and dissemination of scientific research documents, whether they are published or not. The documents may come from teaching and research institutions in France or abroad, or from public or private research centers.
L'archive ouverte pluridisciplinaire HAL, est destinée au dépôt et à la diffusion de documents scientifiques de niveau recherche, publiés ou non, émanant des établissements d'enseignement et de recherche français ou étrangers, des laboratoires publics ou privés. 


\section{Accepted Manuscript}

Title: Réflexions sur le concept de détachement adolescentaire

Authors: M. Delhaye, C. Kempenaers, J. Burton, L. Goossens, P. Linkowski

PII: $\quad$ S0003-4487(10)00241-6

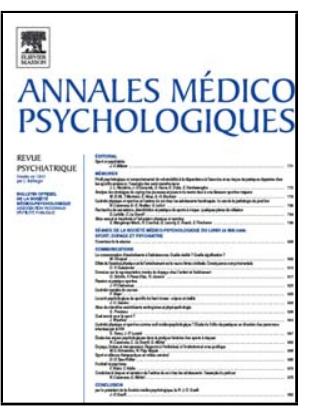

DOI: $\quad$ doi:10.1016/j.amp.2010.07.004

Reference: $\quad$ AMEPSY 1211

To appear in: $\quad$ Annales Médico-Psychologiques

Received date: 23-1-2009

Accepted date: $\quad$ 12-3-2009

Please cite this article as: Delhaye M, Kempenaers C, Burton J, Goossens L, Linkowski P, Réflexions sur le concept de détachement adolescentaire, Annales mediopsychologiques (2010), doi:10.1016/j.amp.2010.07.004

This is a PDF file of an unedited manuscript that has been accepted for publication. As a service to our customers we are providing this early version of the manuscript. The manuscript will undergo copyediting, typesetting, and review of the resulting proof before it is published in its final form. Please note that during the production process errors may be discovered which could affect the content, and all legal disclaimers that apply to the journal pertain. 


\title{
Mémoire
}

\section{Réflexions sur le concept de détachement adolescentaire}

Reflexions about the adolescent detachment concept!

\author{
M. Delhaye ${ }^{\text {a }}$, C. Kempenaers ${ }^{\text {a }}$, J. Burton ${ }^{\text {a }}$, L. Goossens ${ }^{\text {b }}$, P. Linkowski ${ }^{\text {b }}$ \\ ${ }^{a}$ Hôpital Érasme, Université Libre de Bruxelles, 808, Route de Lennik, \\ 1070 Bruxelles, Belgique \\ ${ }^{b}$ Catholic University of Leuven, Department of Psychology, Tiensestraat 102, \\ 3000 Leuven, Belgique
}

Auteur correspondant: Marie Delhaye, Hôpital Érasme, Université Libre de Bruxelles, 808, Route de Lennik, 1070 Bruxelles, Belgique

Adresse email : Marie.Delhaye@erasme.ulb.ac.be

Tél. : 003225553741

Fax : 003225556955

Texte reçu le 23 janvier 2009 ; accepté le 12 mars 2009

\section{Résumé}

Cet article vise à analyser le «détachement adolescentaire » qui est une forme d'autonomisation où la séparation émotionnelle est dysfonctionnelle. Le concept de détachement est décrit selon une vision développementaliste ; l'attachement et le processus de séparation/individuation sont décrits, ainsi que la séparation émotionnelle réussie. Plusieurs types de détachements sont définis en fonction du type d'attachement présenté par le jeune, ainsi que du type de contexte bio-psychosocial dans lequel il s'est déployé. Nous avons donc fait l'hypothèse que le détachement dans une population de jeunes qui présentent des comportements délinquants se réalise sur un mode détaché. Le sujet évite tout ressenti émotionnel. Le détachement chez des jeunes qui sont hospitalisés en pédopsychiatrie se fait quant à lui sur un mode dépressif. Ces adolescents sont incapables de gérer les débordements émotionnels. Et enfin, le détachement dans la population tout-venant peut se faire sur un 
mode plus anxieux. Nous parlons alors de «détachement attaché ». Grâce à une meilleure connaissance du type de séparation que les jeunes mettent en scène, nous pourrons mieux les aider dans la symptomatologie qu'ils présentent. La « clinique du détachement » est née !

Mots clés : Adolescent ; Attachement ; Autonomie ; Détachement ; Famille

\begin{abstract}
This paper aims to analyze the "adolescent detachment" which is a kind of autonomy where the emotional separation is dysfunctional. The detachment concept is described following a developmental view; attachment, separation-individuation and successful emotional separation are described. Several kinds of detachment are defined depending of the attachment type and of the bio-psycho-social context from which the adolescent is. So, we hypothesize that the detachment in an adolescent population with delinquent behaviours is a "detached detachment". The subject avoids any emotional feeling. The detachment for young people who are hospitalised in chid and adolescent psychiatry follows a depressive way. These adolescents cannot handle emotional rush. Finally the detachment for the general young population can evolve according a more anxious way. Then we can talk about an "attached detachment". Thanks to a best knowledge of the separation kind that the adolescents play, we will be able to help them more with the symptoms they show. The "detachment clinic" is born!
\end{abstract}

Keywords: Adolescent; Attachment; Autonomy; Detachment; Family

Comment, au XXI ${ }^{\mathrm{e}}$ siècle, un adolescent peut-il s'autonomiser ? Cet article propose le concept dynamique de «détachement », élaboré à partir d'une revue de la littérature sur le développement de l'autonomisation à l'adolescence et sur ses dysfonctionnements.

Nous proposons une contribution à la réflexion pédopsychiatrique développementaliste actuelle. En effet, un consensus émerge dans la littérature récente : la compréhension des bouleversements liés à l'adolescence se veut avant tout holistique. Ce point de vue amène un éclairage nouveau et fructueux sur le fonctionnement individuel de l'adolescent, ainsi que sur le contexte psychosocial, qui sont étroitement intriqués dès la conception de l'enfant. 


\section{Introduction}

Le détachement est défini comme étant une séparation émotionnelle dysfonctionnelle. Il s'écarte de la séparation normale, saine, et va de pair avec une adaptation psychosociale plus difficile d'accès. Blos (1979) le définit comme une forme extrême de détachement d'avec les parents qui s'accompagne de sentiments d'aliénation et de détresse chez l'adolescent.

Un degré élevé de détachement va de pair avec un sentiment intense d'insécurité par rapport aux parents. Les perceptions se font pessimistes quant aux possibilités pour l'adolescent d'être aimé ; cette insécurité est à mettre en lien avec un attachement difficile. Lamborn et Steinberg [20] situent plus explicitement les sujets dans leur contexte familial ; d'après les auteurs, la signification émotionnelle varie en fonction du type de relation parents/adolescents.

Dans le détachement radical, on implique une série de sentiments négatifs à l'endroit des parents incluant la défiance, l'aliénation et la perception d'une ignorance dans le chef des parents. Tous les items qui mesurent ce détachement ont une connotation péjorative et parfois même légèrement paranoïde [13]. Ce détachement radical, contrairement à la séparation émotionnelle (« saine »), est hautement associé à un conflit répétitif et intense dans la relation de l'adolescent aux parents [3].

On ne peut par ailleurs pas aborder la notion de détachement sans faire un bref rappel de la notion d'attachement.

\section{L'attachement}

La notion d'attachement a d'abord été élaborée en 1887 par R. Spitz à partir de l'observation directe du nourrisson. John Bowlby (1951) a ensuite mis en évidence les mécanismes de la formation et du développement des relations d'attachement. Il a émis le postulat selon lequel l'enfant naît avec un besoin de proximité physique et de contacts sociaux. Ce besoin d'attachement est un besoin primaire vital et inné [6,25].

Mary Ainsworth a étendu la théorie de Bowlby en y associant des données comportementales et une recherche développementale longitudinale, qui ont souligné la stabilité du type d'attachement à travers le temps [3]. La théorie de l'attachement s'est ensuite enrichie de l'apport de la psychanalyse et d'un retour au monde interne suivant le concept de 
«modèle interne opérant », à partir de la représentation interne de la figure d'attachement $[7,9]$.

\subsection{Déploiement de l'attachement pendant la petite enfance}

L'attachement pendant les neuf premiers mois de vie s'organise autour d'un système comportemental qui met en jeu la proximité à la première figure d'attachement (la mère ou la figure maternelle). Ce système a une fonction instinctive essentielle car il régularise la sécurité et promeut la survie (Bowlby, 1969). Lorsqu'il y a un décalage entre la disponibilité attendue de la figure d'attachement et la disponibilité réelle du moment, le système d'attachement est activé. Ce système d'attachement activé permet au nourrisson d'élaborer des tentatives visant à réduire le décalage entre son désir et la réalité. Il va crier, s'agiter, dans le but de restaurer le contact auprès de la figure d'attachement $[7,9]$.

La qualité de l'attachement précoce chez le nourrisson est donc déterminée par les interactions entre la figure d'attachement et l'enfant. Un enfant qui expérimente une figure d'attachement rassurante et répondant à sa détresse est davantage susceptible d'apprendre à réguler son stress. Il utilise des stratégies qui impliquent de pouvoir gérer l'inconfort en cherchant du soutien. Il peut alors développer un «attachement sécure ». L'enfant «attaché » de manière sécure va s'autoriser à explorer l'environnement, la figure d'attachement servant alors de base de sécurité. C'est ce qu'on appelle le «phénomène de base de sécurité » [7]. Les enfants «sécures » sont plus compétents émotionnellement. Ils arrivent à maintenir un comportement structuré dans des situations émotionnellement éprouvantes. Ils présentent des niveaux plus élevés d'empathie [7].

Un enfant qui est confronté à de l'insensibilité ou à de l'inconsistance vis-à-vis des situations de détresse qu'il rencontre est plutôt enclin à associer une conséquence néfaste aux situations de stress. Il peut développer un « attachement insécure » [7].

Quelle que soit la qualité des soins reçus dans la petite enfance (figure soutenante ou au contraire insensible et abusive), l'enfant va acquérir un attachement spécifique [7].

Les stratégies comportementales utilisées pour atteindre plus de sécurisation dépendent à leur tour de la qualité de l'attachement. Mary Ainsworth (1978), au travers de l'expérience de la «Situation Étrange », a défini trois types d'attachement. Cette expérience chez des enfants de 12 mois visait à observer l'attitude de ces derniers pendant et après une courte séparation d'avec la mère $[1,7]$. 
Dans l'«attachement anxieux évitant »(A), l'enfant ignore la mère à son retour et donne une impression d'indépendance affective. Le donneur de soin n'est pas utilisé comme base d'exploration. Les figures d'attachement s'avèrent rejetantes $[1,25]$.

Dans 1 '«attachement sécure » $(\mathrm{B})$, l'enfant proteste mais accueille son parent à son retour avec une expression de soulagement. Les figures d'attachement sont soutenantes (environ deux tiers des sujets selon Cyrulnik) [1,12,25].

Dans l'«attachement anxieux ambivalent»(C), l'enfant est agité. Il se montre ambivalent vis-à-vis du parent avec une tonalité dépendante. Les figures d'attachement sont intrusives et inconsistantes [1,25].

Mary Main (1970) introduit une quatrième catégorie dans les types d'attachement. Il s'agit de l'«attachement anxieux désorganisé » (D). Les stratégies de l'enfant sont mises en échec; l'enfant ne parvient pas à s'approcher du parent, ni à s'en détacher. On rencontre souvent cette problématique dans les situations d'abus, de négligence et de maltraitance $[1,25]$.

Le type d'attachement est en règle générale stable avec le temps, mais des figures d'attachement secondaires vont émerger progressivement avec davantage d'investissements extérieurs ; on intégrera, par exemple, à l'adolescence, les pairs, les intervenants sociaux, les enseignants, la famille élargie. Ce type d'attachement secondaire se vérifie aussi lors du décès d'un parent.

Le modèle interne opérant $[7,12]$ permettra par la suite d'associer la théorie de l'attachement aux représentations internes et aux fantasmes. Fonagy a développé l'idée du « soi réfléchi », qui fait le lien entre le modèle interne opérant et la théorie de l'esprit. L'enfant présente dans ses premières années un fonctionnement mental particulier appelé fonctionnement réflexif. Ce fonctionnement réflexif permet à l'enfant d'interpréter le comportement humain et ses intentions en accord avec ses souhaits, ses craintes et ses attentes. L'enfant perçoit un monde psychique interne, distinct de sa réalité externe. Il se voit par rapport aux autres comme séparé physiquement, mais aussi mentalement. Il présente lui comme l'autre une intentionnalité ; on peut alors parler d'intersubjectivité $[7,12]$.

L'état d'esprit de l'adulte en relation avec ses expériences émotionnelles pendant l'enfance influence ses pratiques parentales (parenting), et ce en maintenant les modèles d'attachement [12]. Cette transmission du style d'attachement s'observe régulièrement. En outre, certains adultes peuvent changer leur style d'attachement en fonction des rencontres qu'ils font avec d'autres figures d'attachement; ils gagnent leur sécurité [7]. 
Le style d'attachement insécure n'est pas prédictif de la survenue d'une psychopathologie ultérieure, il serait davantage un modèle de vulnérabilité qui pourrait induire un trouble ultérieur [12].

\subsection{Attachement et adolescence}

C'est ici qu'intervient l'importance de l'approche développementale qui prend en compte les aspects cognitifs, affectifs, environnementaux, ainsi que les figures d'attachement dans les relations secondaires [7].

Les changements corporels pubertaires rendent les adolescents capables de devenir parents ; l'éveil sexuel remanie les patterns d'attachement. Le jeune «sécure » explore plus facilement son champ relationnel à partir de sa base de sécurité : «Qui suis-je dans la relation avec l'autre ? » La question de la sécurité est cruciale car elle permettra à l'adolescent de développer un intérêt sexuel tout en se protégeant. Le jeune va essayer de trouver une relation où il peut se sentir sexuellement en sécurité dans ses propres limites, tout en prenant un certain nombre de risques pour exprimer ses choix personnels. C'est une tâche complexe qui implique l'influence des parents, des pairs, et celle du contexte social plus large.

L'adolescent retrouve des contacts sensuels dans le rapprochement avec l'autre. Ces contacts lui rappellent ceux de la première enfance au stade de la dyade mère/bébé [7].

Chez les adolescents au style d'attachement anxieux-ambivalent, l'éveil sexuel amène parfois de la confusion avec des sentiments basés sur la peur d'abandon. L'apport d'une réflexion intrapsychique qui s'introduit dans le modèle interne opérant permet de comprendre pourquoi le charme, la séduction et la menace peuvent évoluer comme des caractéristiques centrales au niveau des relations intimes chez ces adolescents. Ils peuvent agresser ceux qu'ils aiment par peur d'être agressés.

Chez les jeunes au style d'attachement anxieux-évitant, le modèle relationnel qui prime est celui de la mise à distance des affects; les adolescents sont plutôt autocentrés. Les sentiments issus de l'intimité sont parfois exclus, déniés de manière défensive. Le sexe sans amour pour faire plaisir à l'autre est expérimenté avec parfois mise en danger et absence de protection [17].

Le type d'attachement va secondairement influencer le choix du partenaire ; le modèle interne opérant de l'un doit être compatible avec le modèle interne opérant de l'autre. Cela implique une certaine flexibilité, car trop de rigidité peut amener à des liaisons physiquement et psychologiquement dangereuses [17]. 
Les styles d'attachement imprégnés dans la mémoire au cours des premiers mois manifestent une discrète tendance à se répéter au cours du premier amour. Les enfants ayant acquis un attachement sécure restent globalement sécures. Cependant, certains vivent ce passage avec angoisse, tandis qu'une minorité éprouve le premier amour comme un traumatisme. À l'inverse, les enfants ayant acquis un attachement insécure peuvent améliorer leur sentiment de sécurité personnelle après le premier amour [8]. Mais plus profonde est l'insécurité de base, plus faible sera le degré d'engagement dans la relation.

Cette analyse nous indique donc que la qualité de l'attachement va influencer la manière par laquelle les adolescents vont se séparer et s'autonomiser.

L'évaluation du style d'attachement à l'adolescence nécessite par conséquent une approche globale bio-psychosociale. Dès la conception, l'enfant va se développer sous l'influence conjointe des facteurs affectifs, cognitifs, biologiques, ainsi que des relations avec son environnement, tout cela dans un processus interactif. Dans cette perspective, l'attachement précoce a une influence sur l'autonomisation de l'adolescent.

Comment se déroule dès lors une autonomisation «normale », une séparation « saine »?

\section{La séparation saine}

\subsection{Séparation-individuation}

Le processus de séparation-individuation commence dans la prime enfance mais se poursuit tout au long du développement et influence inévitablement l'autonomisation $[21,26]$. Le processus de séparation-individuation à l'adolescence n'est pas un processus linéaire dans lequel on passerait d'un état de dépendance à un état d'indépendance ; c'est plutôt un processus où le jeune développe de nouvelles formes d'autonomie de manière discontinue.

Comme dans l'attachement, on s'aperçoit que le processus de séparation-individuation est influencé par l'individu mais aussi par son environnement, et qu'il continuera à être influent dans la vie adulte [18].

Bien que la dynamique de ce processus ait été conçue de manière variée selon les chercheurs, il y a un consensus sur le fait que le jeune adulte doit se différencier des identifications parentales. Il doit établir sa propre voie dans un contexte de relations mutuelles validantes [13]. 
La mutualité des relations parents/adolescents est accomplie lorsqu'il y a un respect bilatéral croissant. Les parents facilitent l'autonomie de l'adolescent dans un contexte de relation proche qui se fait soutenante. Ils communiquent leur fierté dans les compétences croissantes de l'enfant. L'adolescent est encouragé à participer aux prises de décisions familiales [13].

Les garçons doivent « s'écarter » de leur mère et s'identifier à leur père pour trouver une identité masculine. Les filles restent attachées à leur mère pour constituer leur identité féminine [15].

L'individuation est donc au stade de l'adolescence un acquis développemental tardif. L'individuation signifie la séparation intrapsychique par rapport à la famille d'origine [18]. Elle implique une séparation émotionnelle et une différenciation. Les parents sont perçus aussi comme des personnes à part entière, et non plus uniquement comme des parents [2].

\subsection{L'autonomisation}

«L'autonomie est un processus par lequel un individu acquiert ou détermine de luimême ses propres règles de conduite. La capacité d'autonomie résulte de l'intériorisation de règles et de valeurs, consécutive à un processus de négociation personnelle avec les divers systèmes normatifs d'interdépendance et de contraintes sociales.

«D'un point de vue psychodynamique, la conquête d'autonomie est corrélative à la construction de l'identité durant l'adolescence ; elle relève du second processus de séparationindividuation et résulte des remaniements de l'idéal du moi et des identifications ainsi que d'un changement dans l'économie des pulsions ${ }^{1}$. »

Au temps précoce de l'adolescence, l'(ex) enfant est confronté au deuil de l'enfance, un deuil accentué par les transformations corporelles pubertaires. Il est préoccupé par des questions de séparation. En effet, il tente de redéfinir la relation qu'il entretient avec ses parents (objets primaires d'investissement). Un processus de déidéalisation parentale émerge ; ce processus de séparation implique un changement de la représentation du soi. Le jeune va développer une capacité d'introspection associée à un sentiment de solitude [16].

Au milieu de l'adolescence, on assiste en parallèle à un processus d'individuation. Le jeune tente de définir qui il est, ce qui est important pour lui, ce qu'il est capable de faire et ce qui le motive. Il va commencer à s'intéresser aux jeunes du sexe opposé. Un va-et-vient entre le semblable et le différent s'installe. 
À la fin de l'adolescence, le jeune réinvestit les relations familiales. Il est préoccupé par la perspective de se forger des concepts moraux et éthiques. On assiste à une consolidation du processus de séparation-individuation avec un souci de créer des relations formelles, engagées et intimes. Des projets d'avenir sont élaborés. On peut parler de «mature genitality » [16].

L'autonomie à l'adolescence n'est pas un concept unitaire, il peut prendre des formes différentes en fonction du type de relation investie, que ce soit avec les parents, les pairs, ou avec les partenaires amoureux [8]. On distingue dès lors trois types d'autonomie :

1) l'autonomie comportementale ;

2) l'autonomie émotionnelle ;

3) l'autonomie « morale ».

\subsubsection{L'autonomie comportementale}

Elle se réfère aux manifestations actives de fonctionnement qui visent à l'indépendance du sujet, à la régulation de son comportement, ainsi que de ses prises de décisions. Cette autonomie comportementale implique, d'une part, la résolution de conflits «parent/adolescent» où le jeune peut renégocier sa place dans la famille, et, d'autre part, la gestion des prises de décisions concernant son autonomie au regard de l'autorité parentale. Les prises de décisions concertées (parent/jeune) sont les plus positives pour le devenir du jeune. En effet, souvent, les décisions prises unilatéralement par le jeune peuvent entraîner un comportement délinquant, et les décisions unilatérales prises par les parents peuvent contrarier la socialisation [4].

\subsubsection{L'autonomie émotionnelle}

Beyers parle de «Detachment debate»: cette visée, comme on va le voir, est stimulante à travers le contraste qu'elle met au jour entre l'autonomie émotionnelle et le détachement (qui se définit en terme de séparation émotionnelle dysfonctionnelle) [3-5].

Cet auteur se fait principalement l'écho des recherches développementalistes anglosaxonnes (Lamborn SD et Steinberg L, 1993) qui postulent que la séparation émotionnelle est un processus sain [20].

\footnotetext{
1. Ducruet JJ. Dictionnaire de Psychologie. Paris, PUF, 1991.
} 
La « séparation émotionnelle » des sujets jeunes par rapport à leurs parents remplit une fonction d'adaptation pour l'adolescent. C'est un développement « sain » qui est d'abord envisagé. L'autonomie se construit. Le sujet apprend à s'autoréguler, à se forger des valeurs et des principes, et, in fine, à s'affranchir des liens infantiles à la famille qu'il retrouve alors sur un mode plus adulte.

Les futurs adultes réalisent que leurs parents ne sont pas que des parents. Ceux-ci sont perçus « comme d'autres personnes »; ils descendent de leur piédestal (« Désacralisation $\left.{ }^{2} »\right)$. Les jeunes se fondent sur leurs propres ressources, ils deviennent compétents au plan psychosocial [4].

Lamborn et Steinberg [20] sont attentifs à la qualité des relations parents/adolescents ; leur hypothèse est que les jeunes sujets qui ressentent un haut niveau de soutien parental présentent de meilleurs profils d'ajustement au niveau émotionnel. La séparation émotionnelle est facilitée. Ils mettent en évidence « des facteurs qui induisent une adaptation psychosociale chez les adolescents » comme la chaleur humaine, l'implication parentale, la guidance parentale et les faibles niveaux de contrôle autoritaire parental.

Ils font observer que des niveaux élevés d'autonomie émotionnelle chez des enfants de mères émotionnellement stables sont prédictifs de symptômes adolescentaires bas. Ces enfants sont moins anxieux et moins dépressifs, ils ont une estime de soi plus prononcée.

\subsubsection{L'autonomie morale ou cognitive}

Ce type d'autonomie se développe souvent quand les deux autres formes de celle-ci sont internalisées par le jeune. Elle consiste essentiellement en la quête par le jeune de valeurs morales pour poursuivre sa construction identitaire.

\subsection{L'influence du contexte sur l'autonomisation}

Au niveau psychosocial, on assiste à un « détachement-désengagement » dans le cadre sociétal $[10,22]$.

La société s'inscrit de plus en plus dans l'ici et maintenant. Les économies sont en mouvance. Le nomadisme, la précarisation, la rivalité, l'accélération du rythme des changements sont des éléments sociétaux résiduels de la globalisation [10]. Certaines variables renvoient à ce changement sociétal. Parmi celles-ci, on peut citer : la société du 
spectacle et de l'image, l'invasion du virtuel, l'instantanéité, le consumérisme, la fragilisation des structures d'encadrement, l'estompement des normes, le contrôle social moins ferme, la société de l'individu et du narcissisme [22].

Ces éléments contextuels vont intervenir dans l'activation de certains comportements et donc influencer la façon dont on se sépare, dont on s'autonomise, et dont on se détache.

Les comportements vont être plus ou moins contrastés en fonction des expériences rencontrées dans l'environnement. On voit apparaître le repli narcissique, l'évitement, le retrait, la compétition, la rivalité, la non-durée dans les relations, le désengagement, la désappartenance, l'absence de surmoi, l'intolérance à la frustration, l'impulsivité, la rupture, ainsi que le repli sectaire.

Dans ces conditions, un glissement de l'autonomisation vers le détachement peut avoir lieu.

\section{Le détachement}

L'autonomisation de l'adolescent est confrontée par ailleurs à une réorganisation du lien aux parents. Dans la meilleure des hypothèses, le sujet va progressivement pouvoir se positionner dans la société avec ses propres choix, ses propres réflexions et ses propres émotions.

Le détachement, au sens qui lui est donné dans cet article, s'avère plus critique et notamment dans cette réorganisation et ce positionnement sociétal.

Dans les deux concepts développés (l'attachement et la séparation « saine »), on relève la construction psychique progressive de l'autonomisation. Celle-ci implique l'influence de facteurs présents dès les premiers jours de vie. Le futur sujet va s'enrichir au fur et à mesure des contacts avec son environnement. Le tempérament de base de l'enfant va se renforcer suite aux interactions relationnelles, familiales et sociales, au sens large.

Dans le concept de détachement, l'adolescent présente une insécurité de base qui ne lui permet pas d'aborder la séparation du milieu familial de manière positive [3-5,20].

Les relations précoces et le contexte n'ont pas amené les conditions suffisantes pour qu'une autonomisation épanouie puisse s'accomplir. Dès lors, le jeune perçoit les nouvelles relations comme insécurisantes également. Il va donc les aborder de manière spécifique en fonction du type d'attachement, du contexte, et du type de ressources qu'il possède.

\footnotetext{
${ }^{2}$ Désacraliser : dépouiller d'un caractère qui s'imposait jusqu'alors (l'aura parentale).
} 
Le but de cet article est de définir davantage le concept de détachement. La littérature anglo-saxonne est restée cantonnée au détachement en général sans aborder le vécu des jeunes, et ce, dans une population non clinique.

Les auteurs qui se sont penchés sur le problème, grâce à leur pratique clinique, ont vu émerger trois types de détachements différents en fonction de trois populations d'adolescents différentes :

1) le détachement «détaché », qui concernerait les jeunes délinquants ;

2) le détachement «dépressif », qui concernerait les adolescents que l'on retrouve notamment dans les services de pédopsychiatrie ;

3) le détachement «attaché », qui concernerait les jeunes tout-venants qui n'ont pas encore accompli une autonomisation sereine et stable.

\subsection{Détachement détaché}

On retrouve chez ces jeunes des conduites délinquantes (DSM-IV). Bowlby, en 1973, décelait dans ces conduites, une crainte et une menace permanente d'abandon entraînant chez l'enfant un sentiment intense de colère et de rage [7].

Selon Winnicott, la délinquance indique qu'un espoir subsiste. L'adolescent se tourne vers la société plutôt que vers sa famille pour trouver la stabilité dont il a besoin. Cela est encourageant pour le clinicien qui s'intéresse à la thérapie du détachement [7].

Selon notre hypothèse de travail, l'adolescent délinquant a grandi dans une famille où les parents étaient dans le déni des émotions ; ils ne parlaient pas suffisamment à l'enfant. Ces jeunes sont dans un style d'attachement évitant avec un état d'esprit rejetant. Les parents sont absents ou trop coercitifs. Les affects sont inhibés [7].

L'acte délinquant est compris comme une procédure de régulation des affects chez un jeune qui a été soumis à une information sémantique rigide basée sur le mépris des sentiments («pleurer, c'est pour les bébés ») [7].

On observe une instabilité familiale marquée par le chômage, les déménagements fréquents, une discipline rigide avec maltraitance physique et peu de représentations symboliques. Les familles sont parfois monoparentales, ou bien les parents sont porteurs en permanence d'un manque de chaleur envers l'enfant. Le jeune présente peu de motivation scolaire [11].

L'adolescence est pourtant une période de grand développement cognitif avec accès à la pensée formelle (abstraction, raisonnement logico-mathématique) [25], et à la flexibilité. 
De nouveaux outils permettent une réintégration complexe de l'information. L'adolescent se perçoit autrement, ainsi que ses parents, et le monde qui l'entoure. Ce changement de perception peut amener des conflits : «Qui suis-je dans le monde social ? »

La relation aux parents se modifie. L'adolescent a besoin de s'autonomiser tout en recherchant un grand soutien pour arriver à cette autonomie émotionnelle. Il développe des relations en dehors de la famille. L'acquisition de nouvelles fonctions réflexives permet la subjectivation, la différenciation de soi [25], et la recherche de son identité dans un bain de permissivité sélective réciproque avec les parents et la société [19].

L'adolescence est une période de consolidation de l'attachement. Les adolescents sécures arrivent à mieux réguler leurs émotions lors d'un conflit. Les adolescents sécures sont plus compétents socialement que leurs pairs insécures, grâce aux aptitudes émotionnelles qu'ils ont apprises dans leurs relations proches ; aptitudes incluant l'empathie, l'expression et la maîtrise émotionnelle. Un haut niveau d'empathie à l'adolescence a été associé à un comportement prosocial et moins agressif [19].

Les sujets insécures, quant à eux, sont plus enclins à être soit hyperrégulés, soit sousrégulés dans leur expression émotionnelle [19].

Rhode et al. ont trouvé des performances verbales inférieures lors du testing intellectuel chez des sujets aux conduites délinquantes comparés aux autres jeunes de leur âge [27]. Ces jeunes ont connu très tôt le rejet de leurs pairs [11]. Ils développent peu à peu la perception que le monde est dangereux. Ces jeunes ont des capacités d'empathie diminuées $[23,26]$. Les adolescents engagés dans un détachement détaché abordent les relations dans la destructivité. Ils agressent pour ne pas être agressés, ils ne sentent pas dignes d'être aimés puisqu'ils n'ont jamais connu la chaleur, la tendresse dans leurs relations précoces.

\subsection{Détachement dépressif}

Chez ces jeunes, le réseau familial est présent mais fragile. Cela amène un attachement de type insécure ambivalent. Le développement cognitif est limite par rapport aux jeunes de leur âge. Il est envahi par une hyperexpressivité émotionnelle. Le décrochage scolaire temporaire est fréquent. Le débordement émotionnel est mal géré. L'autonomisation est difficile.

Ces jeunes présentent un trouble de l'adaptation (cf. DSM-IV). Ils se retrouvent parfois hospitalisés dans des unités pédopsychiatriques de crise, notamment pour des tentatives de suicide. 
Ces jeunes vivent dans des familles enchevêtrées. Ils restent donc empêtrés dans ce système. L'autonomisation est difficile car elle amène de la culpabilité. Les jeunes ont une difficulté à voir leurs parents comme une «entité séparée ». La relation parents/adolescents est exceptionnellement proche, amenant une grande dépendance chez le jeune du fait du manque de différenciation transgénérationnelle [7]. Une autonomisation entraînerait une trop grande différenciation vis-à-vis des parents ; une différenciation perçue comme dangereuse car elle peut aboutir à l'abandon [7]. L'autonomisation est une menace. L'enfant se construit en «faux-self». Il est habitué à se montrer constamment content. On peut observer ce genre de situation chez les enfants parentifiés. Ceux-ci s'occupent d'un parent faible ou malade. Ils vivent toujours dans la crainte que ce parent disparaisse. Les parents passent alternativement par un comportement très indulgent puis par un comportement rejetant. Une loyauté excessive s'installe chez le jeune vis-à-vis des parents. Il évite les conflits ouverts avec ses parents [13]. Spitz a étudié les effets de la perte des parents avant l'âge de 11 ans, avec comme conséquence, une augmentation de la dépression à l'âge adulte [25].

À l'adolescence, tous les affects enfouis depuis toujours ressortent avec une difficulté de gestion des émotions. La mémoire sémantique est biaisée par une vision trop positive, idéalisée du monde, par opposition à ce que l'on avait perçu dans le détachement détaché où le monde est vécu comme hostile. Les souvenirs autobiographiques (fêtes d'anniversaire par exemple) chez ces jeunes en détachement dépressif sont toujours racontés de manière positive mais avec une incapacité de les détailler [4].

L'adolescent éprouve des difficultés interpersonnelles. L'individuation est douloureuse. Les relations d'objet sont empreintes de peu de différenciation à l'autre. Les représentations parentales sont négatives. Tous ces facteurs peuvent prédisposer à une dépression ultérieure [24]. Ces jeunes vont appréhender les relations avec une angoisse d'abandon importante, menant parfois au rejet des pairs car ils deviennent trop envahissants.

\subsection{Le détachement attaché}

Chez ces adolescents, le réseau familial est très présent. Cela induit un attachement sécure. Les enfants ayant acquis un attachement sécure restent sécures dans l'ensemble. Mais certains vivent ce passage avec angoisse, tandis qu'une minorité éprouve le premier amour comme un traumatisme. On relève un désir de correspondre aux attentes parentales. Le développement cognitif est normal, voire normal supérieur. Les émotions sont exprimées mais contrôlées car ce parenting intensif entraine inévitablement une dette affective. Ces 
jeunes investissent bien le domaine scolaire. L'autonomisation est cependant difficile car ce milieu familial (sur)protecteur a généré de l'anxiété. On retrouve ces adolescents dans la population générale. L'attachement primaire est tellement intense que la séparation est empêchée [24].

Marcel Gauchet attribue cette «impossible entrée dans la vie » au fait que depuis trente ans, on observe un changement des conditions sociales et psychiques dans lesquelles les enfants sont attendus, conçus, et procréés. Ce changement dans le processus d'individualisation contemporain a amené à une désinstitutionalisation de la famille [14]. La famille n'est plus la cellule de base du fonctionnement de la société. Elle est devenue rigoureusement privée. L'enfant aujourd'hui est un enfant du désir. On fait un enfant pour soi et pour lui-même, et non plus pour la perpétuation de la société. Des questions vont alors émerger chez l'adolescent au moment de la constitution de l'identité subjective : «Suis-je vraiment l'enfant qui correspond au désir de mes parents ?» Nul ne peut répondre à cette question. Il s'ensuivra chez le jeune et futur adulte un doute existentiel infini.

Chez l'enfant du désir, l'individuation devient difficile car il éprouve un malaise à se distancier du désir de ses parents. Se poser comme soi implique un détachement vis-à-vis de ceux auxquels on doit la vie. Selon Gauchet, l'enfant désiré fabrique des individus hantés par une aliénation irrévocable à leurs origines. Comment sortir de cette nouvelle famille ? Elle n'est pas faite pour qu'on la quitte puisqu'elle prône les valeurs de reconnaissance du singulier, par opposition à l'impersonnalité de la vie en société [14]. Les adolescents ont parfois peur de s'engager dans des relations car s'engager ce serait quitter son cocon familial.

\section{Conclusion}

Tout cela confirme que l'autonomisation pour l'adolescent d'aujourd'hui est souvent un vrai défi. Ce qui est positif pour les cliniciens qui s'occupent d'adolescents, c'est que l'adolescence est une véritable renaissance. Les jeunes, par leur expression symptomatique, vont remettre en question leur subjectivation pour une meilleure séparation et une meilleure entrée dans la vie adulte.

Ils peuvent se servir de tuteurs de résilience au gré de leurs rencontres (famille, pairs, enseignants, intervenants sociaux). Cette résilience qu'ils ont en propre va leur permettre de mieux faire avec leur expérience du passé. Même si le style d'attachement reste plus ou moins stable, ils vont pouvoir s'en servir comme mécanismes de défense contre les épreuves futures. L'autonomisation devient alors possible. 
Une nouvelle clinique est en train de s'installer, c'est la clinique du détachement. Celle-ci aide le jeune et sa famille à trouver les facteurs de résilience qu'ils ont à l'intérieur d'eux-mêmes, pour que la séparation émotionnelle et l'individuation se fassent le mieux possible.

L'autonomisation à l'adolescence est un processus développemental qui va s'appuyer sur des stades très précoces de la première enfance avec une influence du tempérament propre et des relations avec la famille et l'environnement au sens large dans l'ici et maintenant. Les détachements adolescentaires apparaissent quand la séparation émotionnelle est dysfonctionnelle. L’individuation est alors compliquée.

\section{Conflit d'intérêt : aucun}

\section{Références}

[1] Ainsworth MDS, Blehar MC, Waters E, Wall S. Patterns of attachment: A psychological study of the Strange Situation. Hillsdale, NJ: Erlbaum; 1978.

[2] Andersen B, LaVoie JC, Dunkell CS. Individuation and parents as people: measurement concerns regarding two aspects of autonomy. Journal of Adolescence 2007;30:751-60.

[3] Beyers W. The detachment debate: the separation adjustment link in adolescence. Leuven: Université Catholique de Louvain; 2001.

[4] Beyers W, Goossens L, Vansant I, Moors E. A structural Model of autonomy in Middle and Late Adolescence: Connectedness, Separation, Detachment and Agency. Journal of Youth and Adolescence 2003;32:351-65.

[5] Beyers W, Goossens L, Van Calster B, Duriez B. An alternative Substantive Factor Structure of the Emotional Autonomy Scale. European Journal of Psychological Assessment 2005;21:147-55.

[6] Bowlby J. Attachment and loss, vol 1: Attachment. New York: Basic Books; 1969.

[7] Brown LS, Wright J. Attachment theory in adolescence and its relevance to developmental psychopathology. Clinical Psychology and Psychotherapy 2001;8:15-32.

[8] Cyrulnik B, Delage M, Blein MN, Bourcet S, Dupays A. Modification des styles d'attachement après le premier amour. Ann Méd Psychol 2007;165:154-61.

[9] Delage M. L'enfant et ses figures d'attachement. Journal des professionnels de l'enfance 2008;55:22-5.

[10] De Singly F. Les uns avec les autres. Paris: Armand Colin; 2003. 
[11] Dodge KA, Pettit GS. A Biopsychosocial Model of the Development of chronic conduct problems in Adolescence. Developmental Psychology 2003;39:349-71.

[12] Fonagy P. Théorie de l'attachement et psychanalyse. Paris: Érès; 2004.

[13] Frank SJ, Boige N, Poorman MO, Van Egeren LA, Field DT. Perceived relationships with parents among adolescent inpatients with depressive preoccupations and depressed mood. Journal of Clinical Child Psychology 1997;26:205-15.

[14] Gauchet M. La démocratie contre elle-même. Paris: Gallimard; 2002.

[15] Gnaulati E, Heine BJ. Separation-individuation in late adolescence: an investigation of gender and ethnic differences. Journal of Psychology 2001;135:59-70.

[16] Goossens L, Jackson S. Handbook of adolescent Development. Brussels: Psychology Press; 2002.

[17] Guedeney N., Guedeney A. L'attachement : concepts et applications. Paris: Masson; 2006.

[18] Kroger J. Adolescence as a second separation-individuation process: critical review of an object relations approach. In: E. Skoe and von der Lippe (Eds), Personality development in adolescence: a cross-national and life span perspective. London: Routledge; 1998. p.172-92.

[19] Laible D. Attachment with parents and peers in late adolescence: Links with emotional competence and social behavior. Personality and Individual Differences 2007;43:1185-97.

[20] Lamborn S, Steinberg L. Emotional autonomy redux. Child Dev 1993;64:483-99.

[21] Lapsley DK, Aalsma MC, Varshney NM. A factor analytic and psychometric examination of pathology of separation-individuation. Journal of Clinical Psychology 2001;57:915-32.

[22] Lasch C. La culture du narcissisme. Paris: Climals; 2000.

[23] Lovett BJ, Sheffield RA. Affective empathy deficits in agressive children and adolescents: A critical Review. Clinical Psychology Review 2007;27:1-13.

[24] Milne LC, Lancaster S. Predictors of depression in female adolescents. Adolescence 2001;36:207-23.

[25] Pierrehumbert B. Le premier Lien. Théorie de l'attachement. Paris: Odile Jacob; 2003.

[26] Pine F. Margaret Mahler's concepts of symbiosis and separation-individuation-revisited, re-evaluated, and refined. J of Am Psy Association 2004,52:511-32.

[27] Rohde P, Noell J, Ochs L. IQ scores among homeless older adolescents: characteristics of intellectual performance and associations with psychosocial functioning. Journal of Adolescence 1999;22:319-28. 